\title{
Differential cross-sections at next-to-next-to-leading-order in QCD
}

Charalampos Anastasiou ${ }^{\mathrm{a}}$

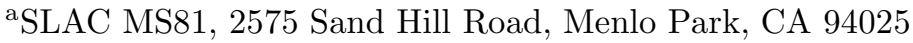

We describe new methods for perturbative calculations and their application to computing next-to-next-toleading-order QCD corrections for Drell-Yan lepton pair production and jet cross-sections.

\section{Introduction}

The Tevatron is at the moment the only collider which probes directly the physics of TeV energy scales. In a few years, the Large Hadron Collider (LHC) will be producing hadron collisions at very high rates and at higher energy. Data from these experiments will provide an excellent opportunity to scrutinize theories against precise measurements.

Recently, new methods have been developed for perturbative calculations. These techniques can be used to produce precise theoretical predictions for observables at collider experiments. Here, we describe the methods that were used in two new computations: the next-to-next-to-leading-order (NNLO) QCD corrections for the rapidity distribution of electroweak bosons at hadron colliders $[1,2]$, and the NNLO QCD corrections for $e^{+} e^{-} \rightarrow 2$ jets $[3,4]$. These are the first differential quantities ever computed at NNLO in QCD.

The first observable was calculated by mapping phase-space integrals onto loop integrals $[5,6]$ and employing multi-loop techniques to reduce their number and evaluate their resulting master integrals. With this method, differential constraints on the phase-space are represented by propagators in a forward scattering amplitude [7]. The constraints are imposed by computing appropriate cuts of the amplitude.

The latter observable was computed by expanding the phase-space integrands in $\epsilon=(4-$ d) $/ 2$, in terms of "delta" and "plus" distributions; this permits the introduction of arbitrary cuts into the phase-space and the generation of partonic events. This $\epsilon$-expansion is possible only after the overlapping singularities are first disentangled $[8,9]$.

In Section 2, we describe the method of mapping phase-space integrations into loop-integrals. In Section 3 we report on results for the rapidity distribution of Drell-Yan lepton pairs at hadron colliders. In Section 4 we describe the method of expanding phase-space in terms of "plus" distributions. Finally, in Section 5 we summarize our discussion.

\section{Mapping phase-space onto loop inte- grals}

To compute a cross-section we must integrate over the momenta of the particles in the finalstate, and over the momenta of particles in loops;

$$
\begin{aligned}
\sigma= & \int \prod_{f}\left[d^{d} q_{f} \Theta\left(q_{f}^{0}\right) \delta\left(q_{f}^{2}-m_{f}^{2}\right)\right] \\
& |\mathcal{M}|^{2} \delta^{d}\left(\sum_{f} q_{f}-\sum_{i} q_{i}\right) \mathcal{O}\left(\left\{q_{f}, q_{i}\right\}\right)(1)
\end{aligned}
$$

Phase-space integrations are constrained; the energy of final-state particles is fixed by a mass-shell condition. Additional constraints are imposed by the observable of interest. These are introduced with a function $\mathcal{O}$ in the integrand. In the evaluation of a total cross-section, $\mathcal{O}=1$. Loop integrations are necessary for the evaluation of the amplitude $\mathcal{M}$, and are simpler than the phase-space integrations since the loop-momenta are unconstrained.

In the last few years there have been significant advances in multiloop calculations. There 
are now exist algorithms [10] to solve integration by parts $[11,12]$ and Lorentz invariance [13] identities, which reduce the number of multiloop computations to a few master integrals. A powerful method to compute the master integrals is the method of Smirnov and Tausk for expanding loop-integrals in $\epsilon$ from their Mellin-Barnes representations $[14,15]$. Another robust approach is to derive and solve differential equations for the master integrals $[13,16,17]$. These methods have led to the computation of two-loop amplitudes for many basic processes at hadron [18-27] and lepton colliders [28-32].

Phase-space integrals pose greater difficulties; however, they can be solved with the above methods after mapping them onto loop-integrals [5]. This is done by replacing all delta functions in Eq. 1 by a propagator-like representation,

$2 \pi i \delta(x)=\lim _{\kappa \rightarrow 0} \frac{1}{x+i \kappa}-\frac{1}{x-i \kappa}$.

We find two types of delta functions: mass-shell constraints $\delta\left(q_{f}^{2}-m_{f}^{2}\right)$, and observable $X$ constraints $\delta\left(X-f\left(\left\{q_{f}\right\}\right)\right)$.

\section{Drell-Yan lepton pair rapidity distribu- tions}

As a concrete example, we consider the rapidity distributions of electroweak gauge bosons $V$ at hadron colliders $[1,2]$. These observables are useful to extract parton densities [33], and monitor the luminosity or partonic luminosities at the LHC [34]. They can probe the electroweak mixing angle [35], and are important for the measurement of the mass of the $W$-boson [36]; both parameters are used to test the self-consistency of the Standard Model and its proposed extensions. Couplings of new gauge bosons to quarks can also be determined at hadron colliders from the rapidity spectrum $[37,38]$.

We study the production of a boson $V$ in the process $h_{1}\left(P_{1}\right)+h_{2}\left(P_{2}\right) \rightarrow V\left(q_{V}\right)+X$. The rapidity $Y$ of $V$ in the rest frame of the two hadrons is defined as: $Y=\frac{1}{2} \ln \left(\frac{q_{V}^{0}+q_{V}^{z}}{q_{\mathrm{V}}^{0}-q_{\mathrm{V}}^{z}}\right)$. The colliding partons carry fractions of the hadronic momenta $p_{1}=x_{1} P_{1}, p_{2}=x_{2} P_{2}$. To compute the rapidity distribution it is sufficient to compute the par- tonic differential distribution in $u=\frac{x_{1} e^{-2 Y}}{x_{2}}=$ $\frac{p_{1} \cdot q_{V}}{p_{2} \cdot q_{V}}$; we write $\mathcal{O}=\delta\left(\frac{q_{V} \cdot\left[p_{1}-u p_{2}\right]}{q_{V} \cdot p_{2}}\right)$ in Eq. 1 . We apply Eq. 2 and replace $\mathcal{O}$ with a "cut" propagator:

$$
\mathcal{O} \rightarrow \frac{q_{V} \cdot p_{2}}{q_{V} \cdot\left[p_{1}-u p_{2}\right]+i \kappa}
$$

Similarly, the mass-shell constraints are replaced by

$\Theta\left(q_{V}^{0}\right) \delta\left(q_{V}^{2}-m_{V}^{2}\right) \rightarrow \frac{1}{q_{V}^{2}-m_{V}^{2}+i \kappa q_{V}^{0}}, \ldots$

converting the phase-space integrations into unrestricted loop-integrations. It is important that we take the limit $\kappa \rightarrow 0$ in order to impose the appropriate "cut" for the propagators that correspond to a constraint. However, this needs only to be done after using integration by parts identities to reduce to master integrals and derive differential equations for the master integrals. The reduction of all integrals was performed using the program AIR [39]. Thirty one master integrals were required, which were calculated by deriving and solving their differential equations.

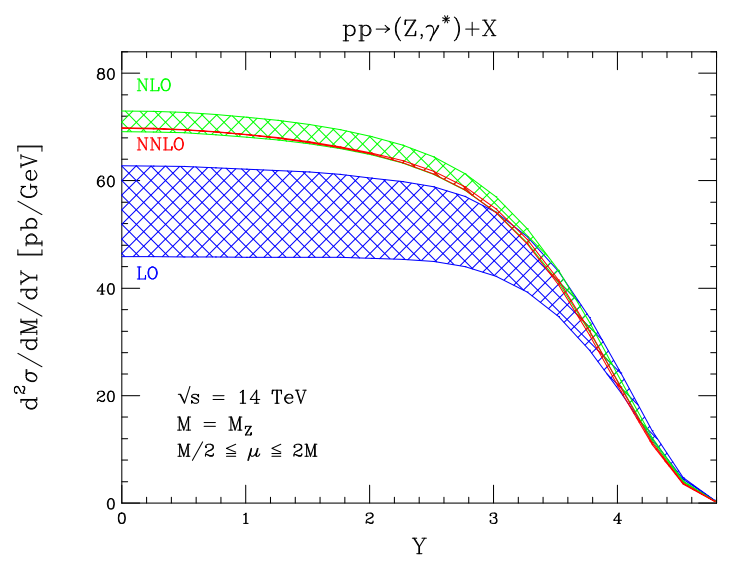

Figure 1. The rapidity distribution of Z-bosons at the LHC

The rapidity distributions of $W$ and $Z$ bosons at NNLO show a remarkable stability with respect to renormalization and factorization scale 
variations for the Tevatron and the LHC [2]. The scale uncertainties drop below $1 \%$ for the experimentally accessible rapidity ranges. At fixed-target experiments [1] the NNLO corrections are quite sizeable. We anticipate that these results will assist in refining parton densities, and strengthen proposals for using the Drell-Yan process as a luminosity monitor at the LHC [34]. It should be noted that higher order electroweak corrections [40-42] are also important at a precision of a few percent.

\section{Exclusive phase-space integrations}

The previous method is useful when the phasespace contains unconsrained variables to integrate over. For some applications fully differential results are needed; for example, to define jets, integrate over the experimentally accepted phasespace, or to merge fixed-order perturbative calculations with parton-shower and hadronization programs [43] we must constrain all final-state variables.

For exclusive phase-space integrations it is necessary to extract the infrared singularities from regions of the phase-space where two or more particles in the final state are not experimentally resolved; these singularities cancel when combined with loop-corrections.

One approach for solving this problem at NNLO in QCD is to subtract from the integrand in Eq. 1 an approximation $\mathcal{F}_{\text {approx }}$ of the squared amplitude in the singular limits [4451]. This function can be multiplied with a simpler observable function $\mathcal{O}_{\text {simple. For example, }}$ $\mathcal{O}_{\text {simple }}$ could allow an inclusive integration over the phase-space of all particles that may become unresolved. The difference

$|\mathcal{M}|^{2} \mathcal{O}-\mathcal{F}_{\text {approx }} \mathcal{O}_{\text {simple }}$

is by construction finite and can be computed numerically. The subtracted term $\mathcal{F}_{\text {approx }} \mathcal{O}_{\text {simple }}$ requires a simpler phase-space integration which can be performed using, for example, the method described in Section 2.

A different method was developed in $[3,4]$. It is always possible to parametrize the phase-space of the final-state particles in Eq. 1 in terms of independent variables $\lambda_{i}$ with $0<\lambda_{i}<1$ :

$$
\begin{aligned}
\sigma & =\int \prod_{f}\left[d^{4-2 \epsilon} q_{f} \Theta\left(q_{f}^{0}\right) \delta\left(q_{f}^{2}-m_{f}^{2}\right)\right]|\mathcal{M}|^{2} \mathcal{O} \ldots \\
& =\int_{0}^{1}\left[\prod_{i} d \lambda_{i}\right] f\left(\epsilon,\left\{\lambda_{i}\right\}\right) \mathcal{O}\left(\left\{\lambda_{i}\right\}\right)
\end{aligned}
$$

The main idea is to expand the function $f\left(\epsilon,\left\{\lambda_{i}\right\}\right)$ as a series in $\epsilon$ in terms of "delta" and "plus" distributions. The coefficients of the $\epsilon$ expansion can be computed numerically for arbitrary observables $\mathcal{O}$.

Terms in $f$ may contain three types of singular structures. The simplest type is a factorized singularity for $\lambda_{i}$ values at the boundaries of the integral, 0 or 1 . For example, if the integrand can be written in the form

$\int_{0}^{1} d \lambda_{k} \lambda_{k}^{-1+\epsilon} g\left(\lambda_{1}, \ldots, \lambda_{k}, \ldots\right) \mathcal{O}\left(\lambda_{1}, \ldots\right)$

where the function $g$ is not singular at $\lambda_{k}=0$ for any of the values of the remaining $\lambda_{i}$ variabes, we can expand in $\epsilon$ using the substitution

$\lambda_{k}^{-1+\epsilon}=\frac{\delta\left(\lambda_{k}\right)}{\epsilon}+\sum_{n=0}^{\infty}\left[\frac{\log \left(\lambda_{k}\right)^{n}}{\lambda_{k}}\right]_{+} \frac{\epsilon^{n}}{n !}$.

A more complicated structure is an overlapping singularity at the boundary, as in the integral

$I=\int_{0}^{1} d \lambda_{1} d \lambda_{2} \frac{\lambda_{1}^{\epsilon} \lambda_{1}^{\epsilon}}{\left(\lambda_{1}+\lambda_{2}\right)^{2}}$.

To disentangle these singularities we divide the integration region into two sectors $[8,9]$ :

$$
\begin{aligned}
& \int_{0}^{1} d \lambda_{1} \int_{0}^{1} d \lambda_{2} \ldots= \\
& \int_{0}^{1} d \lambda_{1} \int_{0}^{\lambda_{1}} d \lambda_{2} \ldots+\int_{0}^{1} d \lambda_{2} \int_{0}^{\lambda_{2}} d \lambda_{1} \ldots
\end{aligned}
$$

By mapping the integration regions back to $[0,1]$, the singularities factorize $[8,9]$; we then apply the expansion of Eq. 7 .

A third type of singularity is a manifold singularity, as in the example integral

$I=\int_{0}^{1} d \lambda_{1} d \lambda_{2} \frac{\left|\lambda_{1}-\lambda_{2}\left(1-\lambda_{1}\right)\right|^{-1+e}}{\lambda_{1} \lambda_{2}}$. 
A singularity occurs on the line of points $\lambda_{1}=$ $\lambda_{1}^{\text {Sing }}=\frac{\lambda_{2}}{1+\lambda_{2}}$ inside the integration region. Note that, as is typical in phase-space integrals, the singular term is explicitly positive definite in the entire integration region. To remove the singularity, we compute the integral in two-sectors: $\lambda_{1}<\lambda_{1}^{\text {Sing }}$ and $\lambda_{1}>\lambda_{1}^{\text {Sing }}$; these cover the entire integration region. The line singularity is, however, moved to the boundaries in each of the two sectors and can be factorized as before.

Manifold singularities also appear in Feynman parametrizations of loop integrals in nonEuclidean kinematic regions (for example, the integral of Eq. 10 without the absolute value). The singular terms are not positive definite in the entire integration region. The singular terms are sign definite, however, in adjacent sectors separated by the manifold of singularities. The complex components of loop integrals become manifest by factoring out the terms $(-1)^{\alpha \epsilon}$, with $\alpha$ integer, which render the singular terms positive definite in all sectors.

This method has been succesfully used to construct a complete program $[3,4]$ for computing fully differential $e^{+} e^{-} \rightarrow 2$ jets observables at NNLO in QCD. This is the first calculation of a fully differential cross-section at NNLO. We anticipate that other NNLO computations can be performed with the same method.

\section{Summary}

We have presented two powerful methods for computing differential cross-sections at higher orders in perturbation theory. The mapping method integrates over variables of the phasespace which are not constrained by the experimental observables. It has been applied for computing cross-sections and differential distributions for hadron collider observables at NNLO in QCD [5-7,1,2]. The method of plus-distribution expansions can be used to compute cross-sections where the experimental observables require constraints on all independent kinematic variables in the phase-space. The first application of the method was the computation of the $e^{+} e^{-} \rightarrow$ 2 jets fully differential cross-section at NNLO in QCD $[3,4]$. Both methods are very general and easily automated; we anticipate that they will be very important theoretical tools for obtaining precise predictions for collider experiments.

Acknowledgements: I am grateful to Lance Dixon, Kirill Melnikov, and Frank Petriello for a very fruitful collaboration. I thank the Fermilab theory group for their hospitality. This research was supported by the US Department of Energy under contract DE-AC03-76SF0515.

\section{REFERENCES}

1. C. Anastasiou, L. J. Dixon, K. Melnikov and F. Petriello, Phys. Rev. Lett. 91, 182002 (2003)

2. C. Anastasiou, L. Dixon, K. Melnikov and F. Petriello, Phys. Rev. D 69, 094008 (2004)

3. C. Anastasiou, K. Melnikov and F. Petriello, Phys. Rev. D 69, 076010 (2004)

4. C. Anastasiou, K. Melnikov and F. Petriello, hep-ph/0402280.

5. C. Anastasiou and K. Melnikov, Nucl. Phys. B 646, 220 (2002)

6. C. Anastasiou and K. Melnikov, Phys. Rev. D 67, 037501 (2003)

7. C. Anastasiou, L. J. Dixon and K. Melnikov, Nucl. Phys. Proc. Suppl. 116, 193 (2003)

8. K. Hepp, Commun. Math. Phys. 2, 301 (1966).

9. T. Binoth and G. Heinrich, Nucl. Phys. B 585, 741 (2000)

10. S. Laporta, Int. J. Mod. Phys. A 15, 5087 (2000)

11. F. V. Tkachov, Phys. Lett. B 100, 65 (1981).

12. K. G. Chetyrkin and F. V. Tkachov, Nucl. Phys. B 192, 159 (1981).

13. T. Gehrmann and E. Remiddi, Nucl. Phys. B 580, 485 (2000)

14. V. A. Smirnov, Phys. Lett. B 460, 397 (1999)

15. J. B. Tausk, Phys. Lett. B 469, 225 (1999)

16. A. V. Kotikov, Phys. Lett. B 254, 158 (1991).

17. Z. Bern, L. J. Dixon and D. A. Kosower, Nucl. Phys. B 412, 751 (1994)

18. C. Anastasiou, E. W. N. Glover, C. Oleari and M. E. Tejeda-Yeomans, Nucl. Phys. B 601, 318 (2001)

19. C. Anastasiou, E. W. N. Glover, C. Oleari and M. E. Tejeda-Yeomans, Nucl. Phys. B 601, 
$341(2001)$

20. C. Anastasiou, E. W. N. Glover, C. Oleari and M. E. Tejeda-Yeomans, Nucl. Phys. B 605, $486(2001)$

21. E. W. N. Glover, C. Oleari and M. E. TejedaYeomans, Nucl. Phys. B 605, 467 (2001)

22. C. Anastasiou, E. W. N. Glover and M. E. Tejeda-Yeomans, Nucl. Phys. B 629, $255(2002)$

23. E. W. N. Glover, JHEP 0404, 021 (2004)

24. E. W. N. Glover and M. E. Tejeda-Yeomans, JHEP 0306, 033 (2003)

25. Z. Bern, A. De Freitas and L. J. Dixon, JHEP 0109, 037 (2001)

26. Z. Bern, A. De Freitas and L. J. Dixon, JHEP 0306, 028 (2003)

27. Z. Bern, A. De Freitas and L. J. Dixon, JHEP 0203, 018 (2002)

28. Z. Bern, L. J. Dixon and A. Ghinculov, Phys. Rev. D 63, 053007 (2001)

29. L. W. Garland, T. Gehrmann, E. W. N. Glover, A. Koukoutsakis and E. Remiddi, Nucl. Phys. B 627, 107 (2002)

30. L. W. Garland, T. Gehrmann, E. W. N. Glover, A. Koukoutsakis and E. Remiddi, Nucl. Phys. B 642, 227 (2002)

31. Z. Bern, A. De Freitas, L. J. Dixon, A. Ghinculov and H. L. Wong, JHEP 0111, 031 (2001)

32. T. Binoth, E. W. N. Glover, P. Marquard and J. J. van der Bij, JHEP 0205, 060 (2002)

33. A. D. Martin, R. G. Roberts, W. J. Stirling and R. S. Thorne, Phys. Lett. B 531, 216 (2002)

34. M. Dittmar, F. Pauss and D. Zurcher, Phys. Rev. D 56, 7284 (1997)

35. F. Abe et al. [CDF Collaboration], Phys. Rev. Lett. 81, 5754 (1998)

36. U. Baur, hep-ph/0304266.

37. F. del Aguila, M. Cvetic and P. Langacker, Phys. Rev. D 48, 969 (1993)

38. M. Dittmar, A. S. Nicollerat and A. Djouadi, Phys. Lett. B 583, 111 (2004)

39. C. Anastasiou and A. Lazopoulos, hep$\mathrm{ph} / 0404258$.

40. U. Baur and D. Wackeroth, hep-ph/0405191.

41. S. Dittmaier and M. Kramer, Phys. Rev. D 65, 073007 (2002)
42. U. Baur and T. Stelzer, Phys. Rev. D 61, 073007 (2000)

43. S. Frixione and B. R. Webber, JHEP 0206, 029 (2002)

44. D. A. Kosower, Phys. Rev. D 67, 116003 (2003)

45. D. A. Kosower, Phys. Rev. Lett. 91, 061602 (2003)

46. D. A. Kosower, hep-ph/0311272.

47. S. Weinzierl, JHEP 0303, 062 (2003)

48. S. Weinzierl, JHEP 0307, 052 (2003)

49. A. Gehrmann-De Ridder, T. Gehrmann and G. Heinrich, Nucl. Phys. B 682, 265 (2004)

50. A. Gehrmann-De Ridder, T. Gehrmann and E. W. N. Glover, hep-ph/0403057.

51. W. B. Kilgore, hep-ph/0403128. 